\title{
Dealing with many correlated covariates in capture-recapture models
}

Olivier Gimenez ${ }^{1}$, Christophe Barbraud ${ }^{2}$

$4{ }^{1}$ CEFE UMR 5175, CNRS, Université de Montpellier, Université Paul-Valéry Montpellier,

5 EPHE, 1919 Route de Mende, 34293 Montpellier Cedex 5, France.

$6 \quad{ }^{2}$ CEBC UMR 7372, CNRS - Université de La Rochelle, 79360 Villiers en Bois, France.

$7 \quad$ Corresponding author: Olivier Gimenez (olivier.gimenez@cefe.cnrs.fr)

$9 \quad$ Word count: 2412

10 Summary: Capture-recapture models for estimating demographic parameters allow covariates

11 to be incorporated to better understand population dynamics. However, high-dimensionality

12 and multicollinearity can hamper estimation and inference. Principal component analysis is

13 incorporated within capture-recapture models and used to reduce the number of predictors

14 into uncorrelated synthetic new variables. Principal components are selected by sequentially

15 assessing their statistical significance. We provide an example on seabird survival to illustrate

16 our approach. Our method requires standard statistical tools, which permits an efficient and

17 easy implementation using standard software.

18 Key words: Animal demography, Population dynamics, Principal-component capture19 recapture model, Survival estimation. 


\section{INTRODUCTION}

Capture-recapture (CR) methods (e.g. Lebreton et al. 1992) are widely used for assessing the effect of explanatory variables on demographic parameters such as survival (Pollock 2002). Generally however, complex situations arise where multiple covariates are required to capture patterns in survival. In such situations, one usually favors a multiple regression-like CR modeling framework that is however hampered by two issues: first, because it increases the number of parameters to be estimated, incorporating many covariates results in a loss of statistical power and a decrease in the precision of parameter estimates; second, correlation among the set of predictors - aka multicollinearity - may alter interpretation (see below).

To overcome these two issues, Grosbois et al. (2008) recommended to perform a principal component analysis (PCA) on the set of explanatory variables before fitting CR models. PCA is a multivariate technique that explains the variability of a set of variables in terms of a reduced set of uncorrelated linear combinations of such variables - aka principal components (PCs) - while maximizing the variance (Jolliffe 2002). Grosbois et al. (2008) then expressed survival as a function of the PCs that explained most of the variance in the set of original covariates, typically the first one or the first two ones.

However, the main drawback of this approach is that the PCs are selected based on covariates variation pattern alone, regardless of the response variable, and without guarantee that survival is most related to these PCs (Graham 2003). To deal with this issue in the context of logistic regression, Aguilera et al. (2006) proposed to test the significance of all

42 PCs to decide which ones should be retained, instead of a priori relying on the PCs that explain most of the variation in the covariates.

In this paper, we implement the algorithm proposed by Aguilera et al. (2006) to deal with many possibly correlated covariates in CR models, a method we refer to as principal 
component capture-recapture (P2CR). We apply this new approach to a case study on survival of Snow petrels (Pagodroma nivea) that is possibly affected by climatic conditions. In this example, the issue of multicollinearity occurs, and summarizing the set of covariates in a subset of lower dimension is also crucial to get precise survival estimates. Overall, $\mathrm{P} 2 \mathrm{CR}$ models can be fitted with statistical programs that perform PCA and CR data analysis. The data and R code are available from GitHub at https://github.com/oliviergimenez/p2cr.

\section{METHODS}

We used capture-recapture (CR) models to study open populations over K capture occasions to estimate the probability $\phi_{i}(i=1, \ldots, \mathrm{K}-1)$ that an individual survives to occasion $i+1$ given that it is alive at time $i$, along with the probability $p_{j}(j=2, \ldots, \mathrm{K})$ that an individual is recaptured at time $j$ - aka as the Cormack-Jolly-Seber (CJS) model (Lebreton et al. 1992). Covariates were incorporated in survival probabilities using a linear-logistic

59 function:

$$
\operatorname{logit}\left(\phi_{i}\right)=\log \left(\frac{\phi_{i}}{1-\phi_{i}}\right)=\alpha+\sum_{j=1}^{p} \beta_{j} X_{i j}
$$

60 where $\alpha$ is the intercept parameter, $X_{i j}$ is the value of covariate $j(j=1, \ldots, p)$ in year $i(i=$

$61 \quad 1, \ldots, \mathrm{K}-1)$, and $\beta_{j}$ is its associated slope parameter. Covariates were standardized to avoid 62 numerical instabilities. To assess the significance of a covariate in CR models, we used the 63 analysis of deviance (ANODEV; Skalski, Hoff \& Smith 1993) that compares the amount of 64 deviance explained by this covariate with the amount of deviance not explained by this 65 covariate, the CR model with fully time-dependent survival serving as a reference. The ANODEV test statistic is given by: 


$$
\operatorname{ANODEV}=\frac{\operatorname{Dev}(X)-\operatorname{Dev}(\text { constant })}{1} / \frac{\operatorname{Dev}(\text { time })-\operatorname{Dev}(X)}{K-1}
$$

where $\operatorname{Dev}($ constant $), \operatorname{Dev}(X)$ and $\operatorname{Dev}($ time $)$ stand for the deviance of models with constant, covariate-dependent and time-dependent survival probabilities. To obtain the associated pvalue, the value of the ANODEV is compared with the quantile of Fisher-Snedecor distribution with 1 and K-1 degrees of freedom.

To reduce the dimension of the set of covariates $\left(\mathrm{X}_{1}, \ldots, \mathrm{X}_{\mathrm{p}}\right)$, we used PCA which aims at finding a small number of linear combinations of the original variables - the principal components $(\mathrm{PCs})$ - while maximizing the variance in $\left(\mathrm{X}_{1}, \ldots, \mathrm{X}_{\mathrm{p}}\right)$. Because the variables measurement units often differ, we performed the PCA on the correlation matrix (Jolliffe 2002). To select PCs, we used a forward model selection algorithm as proposed by Aguilera et al. (2006) for the logistic regression. The forward algorithm begins with no covariates in the model. Each PC is incorporated in simple linear regression-like CR models and the ANODEV p-value calculated. The PC that has the lowest p-value is added to the null model, say $\mathrm{PC}_{\mathrm{k}}$. Then the PCs that were not retained are incorporated along with $\mathrm{PC}_{\mathrm{k}}$ in multiple regression-like CR models, and ANODEV p-values are calculated. In other words, we need to assess the effect of $\mathrm{PC}_{\mathrm{j}}$ for $\mathrm{j} \neq \mathrm{k}$ in the presence of $\mathrm{PC}_{\mathrm{k}}$ to decide whether $\mathrm{PC}_{\mathrm{j}}$ should be retained. To do so, $\operatorname{Dev}($ constant $)$ and $\operatorname{Dev}(X)$ are replaced by $\operatorname{Dev}\left(\mathrm{PC}_{\mathrm{k}}\right)$ and $\operatorname{Dev}\left(\mathrm{PC}_{\mathrm{k}}+\mathrm{PC}_{\mathrm{j}}\right)$ in Equation 2, where $\operatorname{Dev}\left(\mathrm{PC}_{\mathrm{k}}+\mathrm{PC}_{\mathrm{j}}\right)$ is the deviance of the model with survival as a function of both principal components $\mathrm{PC}_{\mathrm{k}}$ and $\mathrm{PC}_{\mathrm{j}}$. We repeat the process until no remaining $\mathrm{PC}$ is selected.

All models were fitted using the maximum-likelihood method using MARK (White \& Burnham 1999) called with R (Laake 2013). 
93 The Snow petrel is a medium sized Procellariiform species endemic to Antarctica that breeds

94 in summer. Birds start to occupy breeding sites in early November, laying occurs in early

95 December and chicks fledge in early March. This highly specialized species only forages

96 within the pack-ice on crustaceans and fishes. Data on survival were obtained from a long-

97 term CR study on Ile des Pétrels, Pointe Géologie Archipelago, Terre Adélie, Antarctica. We

98 refer to Barbraud et al. (2000) for more details about data collection. We removed the first

99 capture to limit heterogeneity among individuals, and worked with a total of 604 female

$100 \quad$ capture histories from 1973 to 2002.

The following covariates were included to assess the effect of climatic conditions

102 upon survival variation: sea ice extent (SIE; http://nsidc.org/data/seaice_index/); air

103 temperature, which was obtained from the Météo France weather station at Dumont

104 d'Urville, as a proxy for sea surface temperature; southern Oscillation Index (SOI) as a proxy

105 for the overall climate condition (https://crudata.uea.ac.uk/cru/data/soi/). These

106 environmental variables were averaged over seasonal time periods corresponding to the chick

107 rearing period (January to March: summer period), the non-breeding period (April to June:

108 autumn and July to September: winter), and the laying and incubation period of the same year

109 (October to December: spring). In total, 9 covariates were included in the analysis: sea ice

110 extent in summer (SIEsummer), in autumn (SIEautumn), in winter (SIEwinter), in spring

111 (SIEspring), annual SOI, air temperature in summer (Tsummer), in autumn (Tautumn), in 112 winter (Twinter) and in spring (Tspring). 
115 The CJS model poorly fitted the data $\left(\chi^{2}=221.2, \mathrm{df}=127, \mathrm{p}<<0.01\right)$, and a closer

116 inspection of the results revealed that the lack of fit was explained by a trap-dependence

117 effect $\left(\right.$ Test2CT, $\left.\chi^{2}=103.1, \mathrm{df}=27, \mathrm{p}<<0.01\right)$. Consequently, we estimated two recapture

118 probabilities that differed according to whether or not a recapture occurred the occasion

119 before (Pradel 1993). By first attempting to simplify the structure of recapture probabilities,

120 we were led to consider an additive effect of time and a trap effect (Supplementary material).

121 Estimates of recapture probabilities ranged from 0.14 (standard error $[\mathrm{SE}]=0.07)$ to 0.79 (SE

$122=0.09)$ when no recapture occurred the occasion before and from $0.25(\mathrm{SE}=0.18)$ to 0.89

$123(\mathrm{SE}=0.09)$ when a recapture occurred the occasion before (Supplementary material).

124 Because of multicollinearity, we were led to counterintuitive estimates of regression

125 parameters in the CR model including all covariates (Supplementary material): the coefficient

126 of SIE in autumn was estimated at $0.5(\mathrm{SE}=0.24)$ and that of SIE in winter was estimated at

$127-0.5(\mathrm{SE}=0.21)$ while these two covariates were significantly positively correlated $(\mathrm{r}=0.67$,

$128 \mathrm{p}<0.01)$.

129 When we applied the P2CR approach, the algorithm selected two PCs, namely PC3

$130 \quad\left(\mathrm{~F}_{1,27}=7.34, \mathrm{p}=0.01\right)$ at step 1 and PC4 $\left(\mathrm{F}_{1,26}=4.63, \mathrm{p}=0.04\right)$ at step 2 (Supplementary

131 material), but never did we pick PC1 as we would have done using a classical approach

132 (Grosbois et al. 2008). PC3 was positively correlated to SIE in summer and negatively

133 correlated to temperature in winter, while PC4 was positively correlated to temperature in

134 spring and negatively correlated to SIE in summer (Supplementary material). Survival

135 increased with increasing values of PC3 (Figure 1), with high values of SIE in summer and

136 low values of temperature in winter (resp. low values of SIE in summer and high values of

137 temperature in winter) corresponding to high (resp. low) survival. 


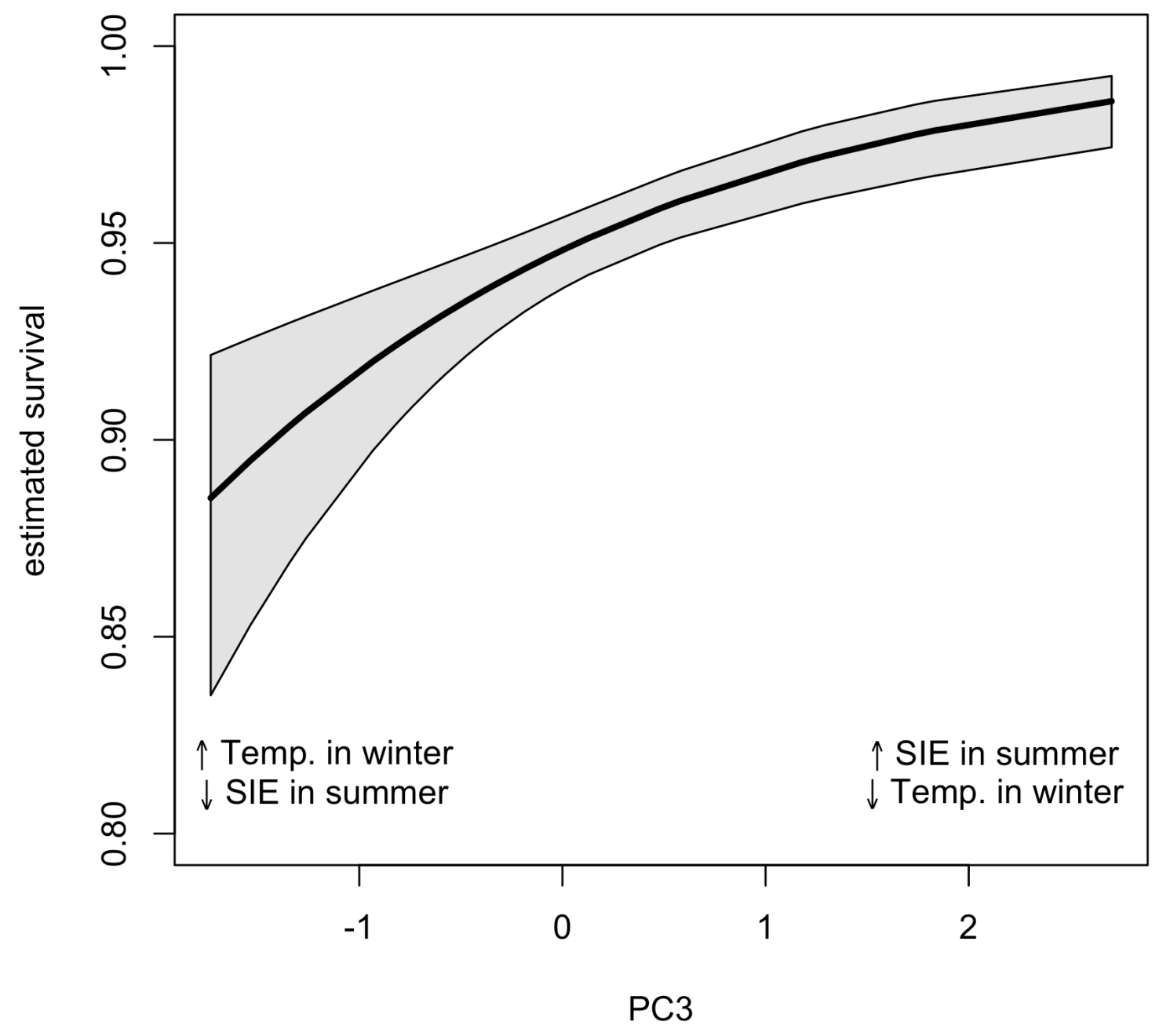

141 Survival decreased with increasing values of PC4 (Figure 2), with high values of temperature

142 in spring and low values of SIE in summer (resp. low values of temperature in spring and

143 high values of SIE in summer) corresponding to low (resp. high) survival. 


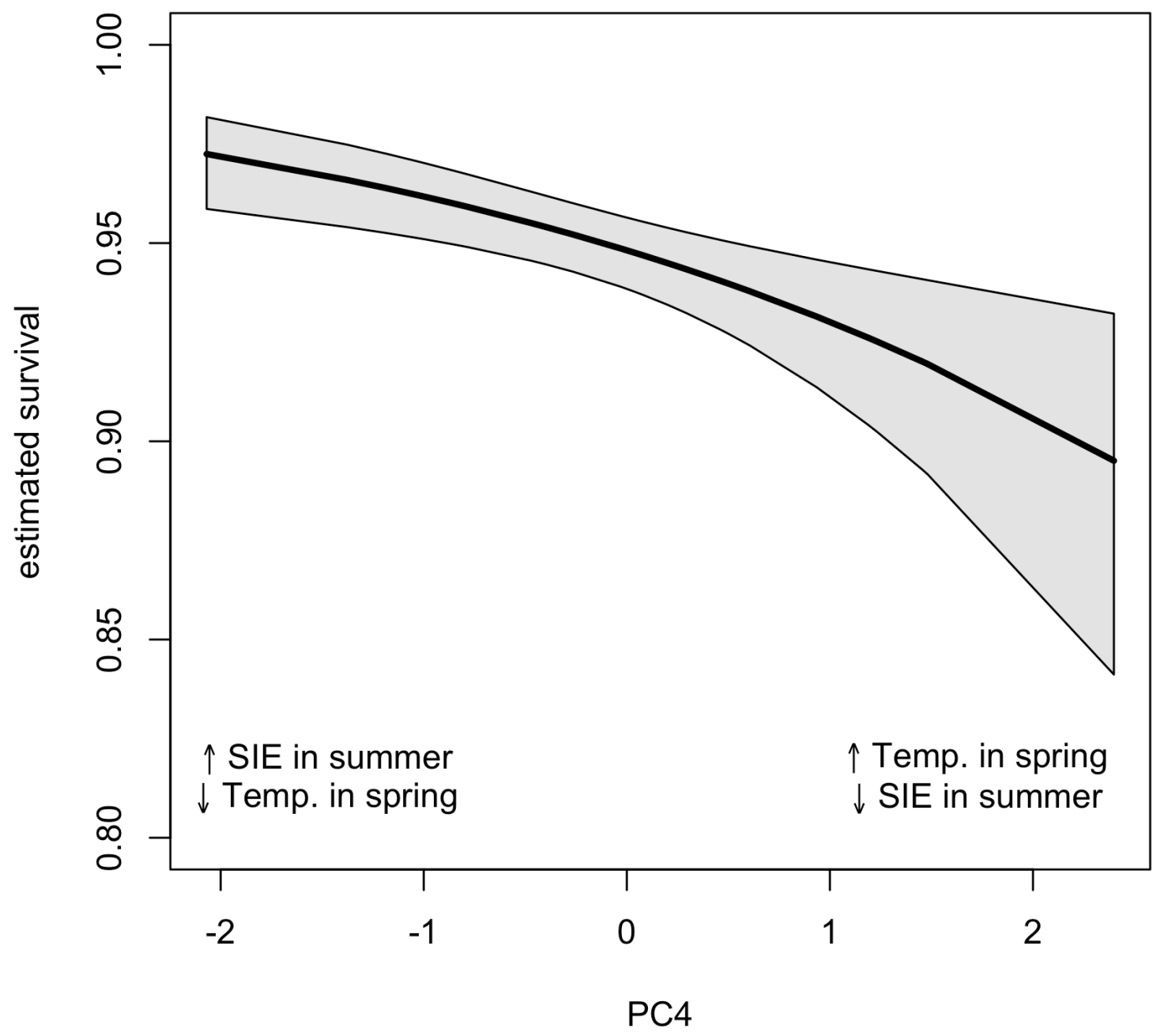

148 The P2CR approach also led to more precise survival estimates when compared to the model

149 incorporating all original covariates (Figure 3). 

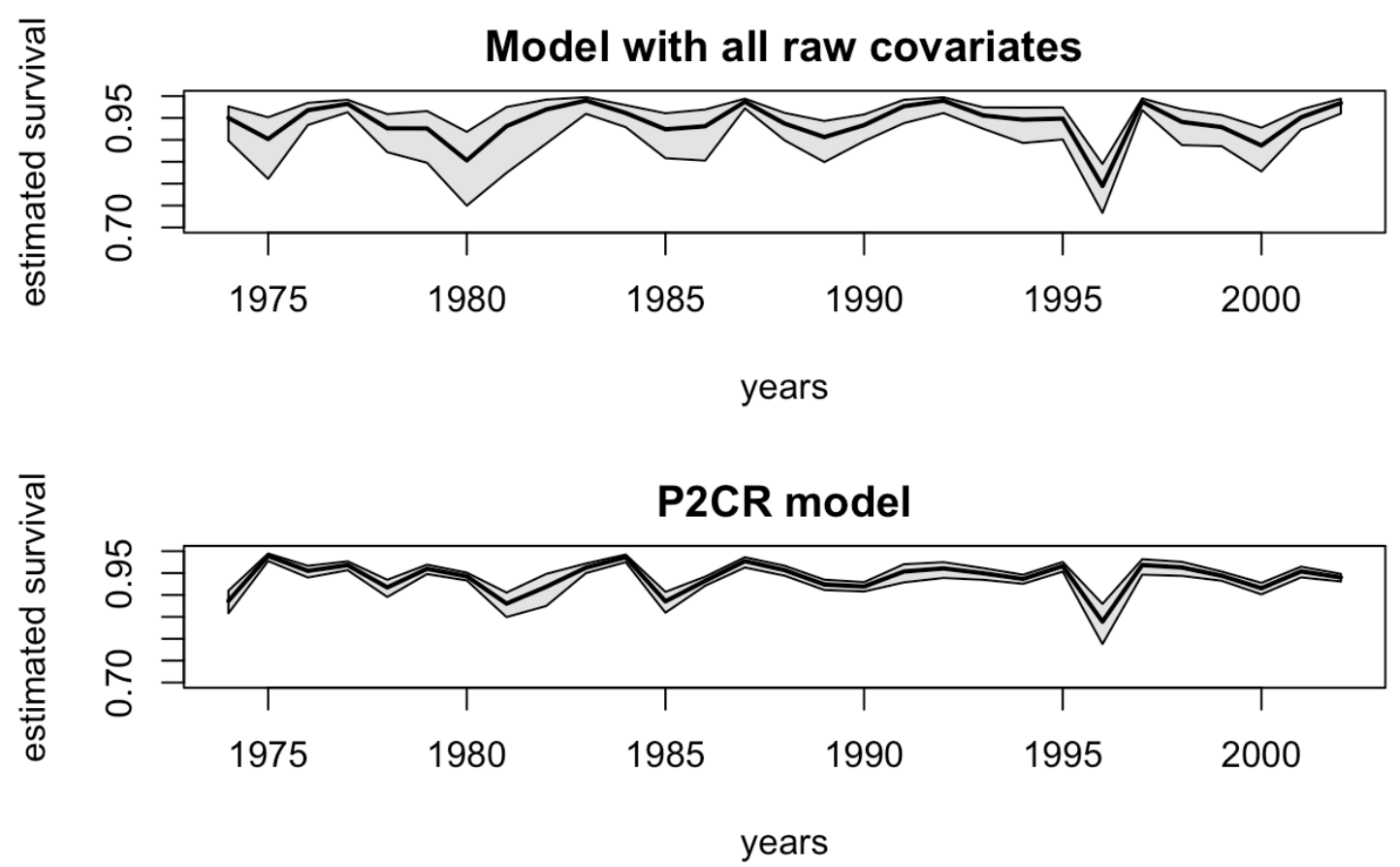

Figure 3: Survival of Snow petrel over time as estimated from the model with all original covariates (top panel) vs. the PC2R model (bottom panel).

We introduce a new approach combining principal component analysis and capture-recapture models to deal with many possibly correlated explanatory covariates. Our approach requires standard statistical tools, which allows an efficient and easy implementation using standard software.

164 In summer, snow petrels exclusively forage within the pack-ice tens to hundreds of

165 kilometers from the colony where they catch sea ice-associated species, such as Antarctic

166 silverfish (Pleuragramma antarcticum) and Euphausiids, to feed their chick (Ridoux \&

167 Offredo 1989). This is an energetically demanding period for breeding adults and, during 
168 years with reduced sea-ice extent, food resources may be less abundant and snow petrels may

169 be forced to cover larger distances to find suitable foraging habitats, with potential survival

170 costs. Assuming air temperature was a proxy of sea surface temperature variations, the

171 negative effect of warmer temperatures on survival is coherent with general patterns found

172 between sea surface temperature and demographic parameters in seabirds (Barbraud et al.

173 2012). In many marine ecosystems warmer temperatures are associated with decreased

174 primary production and food resources for top predators. Although the low survival in 1996

175 corresponded to a year with reduced sea-ice extent in summer, the drop in survival was high

176 and remains unexplained at the moment.

179 When multiple covariates have to be considered to estimate survival, both issues of

180 dimensionality and multicollinearity can lead to biased estimates, inflated precision as well as

181 lack of statistical power. In such a context, the P2CR modeling framework has proved

182 particularly useful in our example, mainly because few PCs were selected which were easily

183 interpretable. We acknowledge that PCs with little interpretability might have been picked up

184 by our method. To make the interpretation easier, PCA results can be post-processed by

185 rotating axes to improve correlations between raw variables and PCs like in the varimax

186 method (Kaiser 1958). Recent developments in the field of multivariate analyses could also

187 be useful, like methods to handle with missing values in PCA (Dray \& Josse 2015).

188 In statistical ecology, one of our objectives is to try and explain variation in state

189 variables such as abundance, survival and the distribution of species. Dimension-reduction

190 methods are promising to deal with many correlated covariates for the analysis of CR or

191 occupancy data. 


\section{ACKNOWLEDGEMENTS}

We greatly acknowledge all of the wintering fieldworkers involved in the monitoring programs in Terre Adélie since 1962, and Dominique Besson for the management of the database. The study on petrels was supported by Expéditions Polaires Françaises, Institut Paul Emile Victor (program IPEV 109, resp. H. Weimerskirch) and Terres Australes et Antarctiques Françaises.

\section{LITERATURE CITED}

Aguilera, A.M., Escabias, M. \& Valderrama, M.J. (2006) Using principal components for estimating logistic regression with high-dimensional multicollinear data. Computational Statistics and Data Analysis, 50, 1905-1924.

Barbraud, C., Rolland, V., Jenouvrier, S., Nevoux, M., Delord, K. \& Weimerskirch, H. review. Marine Ecology Progress Series, 454, 285-307.

Barbraud, C., Weimerskirch, H., Guinet, C. \& Jouventin, P. (2000) Effect of sea-ice extent on

Dray, S. \& Josse, J. (2015) Principal component analysis with missing values: a comparative survey of methods. Plant Ecology, 216, 657-667.

212 Graham, M.H. (2003) Confronting multicollinearity in ecological multiple regression. Ecology, 84, 2809-2815.

214 Grosbois, V., Gimenez, O., Gaillard, J.M., Pradel, R., Barbraud, C., Clobert, J., Møller, A.P. 215 \& Weimerskirch, H. (2008) Assessing the impact of climate variation on survival in vertebrate populations. Biological Reviews, 83, 357-99.

217 Jolliffe, I.T. (2002) Principal Component Analysis, Second Edition. Springer-Verlag, New 
York.

219 Kaiser, H.F. (1958) The varimax criterion for analytic rotation in factor analysis.

Laake, J.L. (2013) RMark: An R Interface for Analysis of Capture-Recapture Data with MARK. AFSC Processed Rep 2013-01, 25p. Alaska Fish. Sci. Cent., NOAA, Natl. Mar. Fish. Serv., 7600 Sand Point Way NE, Seattle WA 98115.

Lebreton, J.-D., Burnham, K.P., Clobert, J. \& Anderson, D.R. (1992) Modeling survival and testing biological hypotheses using marked animals: A unified approach with case studies. Ecological Monographs, 62, 67-118.

Pollock, K.H. (2002) The use of auxiliary variables in capture-recapture modelling: an overview. Journal of Applied Statistics, 29, 85-102.

Ridoux, V. \& Offredo, C. (1989) The diets of five summer breeding seabirds in Adélie Land, Antarctica. Polar Biology, 9, 137-145.

Skalski, J.R., Hoff, A. \& Smith, S.G. (1993) Testing the significance of individual- and cohort-level covariates in animal survival studies. Marked Individuals in the Study of

234 White, G.C. \& Burnham, K.P. (1999) Program MARK: survival estimation from populations of marked animals. Bird Study, 46, 120-139. 